\title{
Funcionamiento social en niños hemofílicos. Análisis de encuesta para determinar factores psicopatológicos de riesgo
}

\author{
Sandra Sarmiento ${ }^{1 a}$, Cecilia Carruyo de Vizcaíno ${ }^{2 b}$, \\ Edgardo Carrizo ${ }^{3}$, Jennifer Vizcaíno $C^{4 c}$, \\ Melvis Arteaga-Vizcaíno ${ }^{3}$, G ilberto Vizcaíno3. \\ Social functioning and \\ psychopathologic risk factors in \\ hemophilic children
}

Background: Children with hemophilia can experience problems with their mental health status and social functioning. Aim: To assess the mental health status of hemophilic children. Material and methods: Thirty four hemophilic children aged 5 to 13 years were studied. A translation of the special survey to assess mental health in children, denominated "Domingo" Mental Health Survey was applied. It consisted in animated cartoon questions related to the family, social and school life. According to the score obtained, children were classified as normal, doubtful or pathologic. The social functioning areas studied were family, school, relationship and emotional. Aggressiveness, depression/anxiety and rejection were the psychopathologic factors analyzed. Results: Forty four percent of hemophilic children were considered normal, $20 \%$ doubtful and 35\% pathologic. According to the severity of the disease, $67 \%$ of children with severe, $57 \%$ with moderate and $45 \%$ with mild hemophilia, were considered abnormal. Aggressiveness was the main risk factor in the emotional and family area, depression/ anxiety in the family and emotional area and rejection in the family area. Fifty six percent of children had abnormalities in their social functioning and the severity of the disease was a predisposing factor. Conclusions: Psychopathologic factors in children with hemophilia appear mainly in the family environment (Rev Méd Chile 2006; 134: 53-9).

(Key words: Hemophilia A; Hemophilia B; Mental health; Psychological phenomena and processes)

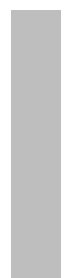

\footnotetext{
Recibido el 28 de octubre, 2004. Aceptado el 7 de julio, 2005.

${ }^{1}$ Banco de Sangre del Estado Zulia, Venezuela. ${ }^{2}$ Sección de Orientación, División de Educación Médica, Facultad de Medicina, Universidad del Zulia, Venezuela. ${ }^{3}$ Instituto de Investigaciones Clínicas, Facultad de Medicina, Universidad del Zulia, Venezuela. ${ }^{4}$ Hospital Materno Infantil Cuatricentenario, Ministerio de Salud y Desarrollo Social, Maracaibo, Venezuela. aResidente Hematología

${ }^{\mathrm{b}} \mathrm{MgS}$ en Orientación de la Conducta

cResidente de Pediatría
}

Correspondencia a: Gilberto Vizcaíno. Instituto de Investigaciones Clínicas, Facultad de Medicina, Universidad del Zulia, Maracaibo-Venezuela. Zona Postal 4001-A. Teléfono: 58-2617597248. Fax: 58-2617-597247. E-mail: g_vizcaino@hotmail.com 
$\mathrm{L}$ a hemofilia es una enfermedad de la coagulación, hereditaria, crónica, ligada al sexo, cuya evolución está caracterizada por las complicaciones ocasionadas por hemorragias recurrentes que ocasionan discapacidad física, que depende de la gravedad de la enfermedad. Las secuelas y complicaciones de la hemofilia son producto del manejo inoportuno del tratamiento en calidad, cantidad y seguridad biológica, causando una gran carga psíquica y física de carácter invalidante y riesgo de muerte.

La Federación Mundial de la Hemofilia ${ }^{1}$ señala que el desafío actual es ayudar a prestar un cuidado integral a todas las familias con hemofilia, la meta central es, además de aplicar el tratamiento sustitutivo adecuado para evitar secuelas, restaurar su sensación de bienestar, su derecho a percibirse como persona, parte de una familia, de un grupo de amigos, con talento y capacidad para el desarrollo, aun cuando presente una enfermedad que le impone limitaciones y cuidados.

El cuidado de los niños con hemofilia en países desarrollados, está dirigido a enfatizar la salud del niño, no la enfermedad, lo cual es abordado a través de programas integrales de tratamiento destinados a obtener calidad de vida ${ }^{2}$. El conocimiento del estado de salud mental y de los factores psicopatológicos de funcionamiento social de los niños con hemofilia, es de vital importancia para el desarrollo y adaptación de ellos a su ambiente familiar, escolar y social.

Los aspectos psicosociales de la hemofilia han recibido una menor atención en comparación con la sintomatología clínica. En relación con el funcionamiento social, los niños hemofílicos pueden presentar alteraciones de adaptación social ocasionadas probablemente por el grado de severidad de la hemofilia, por la relación con el núcleo familiar (familia dividida), por trastornos emocionales o por poca integración con sus compañeros de escuela. No obstante algunas publicaciones señalan que los niños hemofílicos pueden compararse con sus pares normales o con otras afecciones hematológicas hereditarias, en casi todas las áreas, mostrando una adaptación social adecuada, debida probablemente a una mejor atención integral del niño ${ }^{3}$. Sin embargo, en ocasiones reportan dificultades en el bienestar emocional, incluyendo sintomatología depresiva y baja autoestima ${ }^{4}$. Otras publicaciones mencionan que la ansiedad por el riesgo a sangrar, el miedo a infecciones transmitidas por productos sanguíneos y la tendencia a la depresión, afectan no sólo al niño hemofílico sino también a sus padres ${ }^{5}$.

La calidad de vida en el niño hemofílico ha emergido como la primera medida en resultados de salud 6 , porque permite la interacción de los indicadores tradicionales obtenidos desde la percepción del hemofílico, un ejemplo de ello es la disyuntiva si el tratamiento profiláctico es superior al tratamiento por demanda en lograr una mejoría en los síntomas dolorosos, actividad física, salud mental y funcionamiento social, aparentemente la balanza se inclina hacia la primera opción de tratamiento 7,8 .

Un estudio reciente ha explorado los mecanismos de asociación entre los episodios hemorrágicos en niños hemofílicos y su rendimiento académico ${ }^{9}$, encontrándose que el ausentismo escolar está en relación directa con el número de episodios hemorágicos y con un menor rendimiento académico.

La presente investigación estableció como objetivo determinar el estado de salud mental de los niños hemofílicos, mediante la aplicación y análisis de una encuesta programada para obtener respuestas sobre funcionamiento social, con base en la detección de factores psicopatológicos de riesgo relativos al ambiente psicosocial.

\section{MATERIAL Y MÉTODO}

Se realizó una investigación de carácter prospectiva, descriptiva y analítica, mediante la aplicación de un instrumento denominado Encuesta de Salud Mental «omingo» (ESMD), a 34 niños hemofílicos con edades comprendidas entre los 5 y 13 años que asisten al Instituto Hematológico de Occidente-Banco de Sangre del Estado Zulia en la ciudad de Maracaibo, al occidente de Venezuela. Treinta de ellos estaban clasificados como hemofilia A y 4 como hemofilia B. Según el grado de afectación clínica, 9 eran hemofilia severa $(<1 \%$ del factor deficiente), 14 hemofilia moderada (de 1 a $5 \%$ de factor deficiente) y 11 hemofilia leve (>de $5 \%$ del factor deficiente).

Se obtuvo el consentimiento informado con la aprobación de los representantes de los niños participantes en el estudio, así como del Comité de Ética de la Institución de Salud Pública donde se atienden los pacientes con diagnóstico de hemofilia. 
A cada uno de ellos se les aplicó la ESMD, instrumento diseñado por Jean Pierre Vallás en Québec (Canadá) y traducida y validada al español en el Instituto Nacional de Psiquiatría Infantil (INAPSI), Caracas, Venezuela ${ }^{10}$. Se caracteriza por ser una encuesta de pesquisa y es un diseño instrumental para la evaluación de la salud mental en la población infantil. La ESMD está conformada por una especie de tira cómica que está destinada a minimizar o hacer desaparecer los inconvenientes que presentan los auto-cuestionarios.

La técnica de la ESMD conduce a plantear una pregunta al niño con la ayuda de una imagen, en donde la introducción de dibujos que muestran niños felices en situación normal, tiene la ventaja de hacer más aceptable el instrumento y ocasionar menos respuestas patológicas. En el caso de la detección del factor agresividad, se ha representado por ejemplo la oposición hacia sus padres, la agresividad del niño solo y hacia los iguales. Un niño normal responderá positivamente a algunos de estos dibujos, mientras que el agresivo responderá positivamente a la mayoría de ellos. Así, la ESMD explora la forma en la que el niño considera su propia vida, es decir su mundo interior. La puntuación se obtiene reagrupando el conjunto de respuestas a las diferentes láminas de ESMD. Se consideró para este puntaje como iguales 1, todas las respuestas positivas y cero las negativas de un niño con salud mental ideal. Sumando las respuestas así reordenadas, se obtiene para cada niño una puntuación total. Según la puntuación general obtenida se consideran tres tipos de categorías: 1) Categoría patológica, los que obtuvieron 13 y más puntos. 2) Categoría dudosa

\section{Tabla 1. Identificación de las categorías de salud mental en los niños con hemofilia, según la encuesta de salud mental «D omingo»}

\begin{tabular}{|lrc|}
\hline Categorías & n Casos & Porcentaje \\
\hline Normal & 15 & $44,12 \%$ \\
Dudoso & 7 & $20,59 \%$ \\
Patológico & 12 & $35,29 \%$ \\
Total & 34 & $100,00 \%$ \\
\hline
\end{tabular}

(puntuación entre 10 a 12) y 3) categoría normal, con puntuación entre 0 y 9 . Los factores de funcionamiento social psicopatológicos, fueron identificados en cada categoría como: agresividad, depresión-ansiedad y rechazo por los iguales.

Los resultados se analizaron y se expresaron en valores absolutos y porcentuales.

\section{RESULTADOS}

Los datos obtenidos al aplicar la Encuesta de Salud Mental «omingo» (ESMD) sobre la salud mental en niños hemofílicos, mostraron que $44,1 \%$ (15/34 pacientes) se ubicaron en la categoría normal, 20,6\% (7/34 pacientes) en la categoría dudoso y $35,3 \%$ (12/34 pacientes) en la categoría patológico (Tabla 1).

Al relacionar el grado clínico de la hemofilia con las categorías de salud mental (Tabla 2), se encontró que en la forma severa de la hemofilia, $55,5 \%$ pertenecían a la categoría patológico, en las

Tabla 2. Relación del grado de afectación clínica de la hemofilia y los estados de salud mental según la encuesta «D omingo»

\begin{tabular}{|cccc|}
\hline $\begin{array}{c}\text { Grado clínico de } \\
\text { la hemofilia }\end{array}$ & Normal (\%) & $\begin{array}{c}\text { Categorías de salud mental } \\
\text { Dudoso (\%) }\end{array}$ & Patológico (\%) \\
\hline $\begin{array}{c}\text { Hemofilia leve } \\
(11)\end{array}$ & $6(54,5)$ & $1(9,1)$ & $4(36,4)$ \\
$\begin{array}{c}\text { Hemofilia moderada } \\
(14)\end{array}$ & $6(42,9)$ & $5(35,7)$ & $3(21,4)$ \\
$\begin{array}{c}\text { Hemofilia severa } \\
(9) \\
\text { Total } \\
(34)\end{array}$ & $3(33,3)$ & $1(11,1)$ & $5(55,5)$ \\
\hline
\end{tabular}


formas de hemofilia moderada y leve la mayoría de ellos se agruparon en la categoría normal $(42,9 \%$ y $54,5 \%$, respectivamente).

La Figura 1, representa los porcentajes de las áreas de funcionamiento social con las cuales se identifica el niño hemofílico según la categoría de salud mental. Se observa que los casos ubicados en la categoría patológico presentan relación con casi todas las áreas de funcionamiento social. Se muestra además, una disminución porcentual progresiva del área familiar entre los normales y los patológicos con incremento en las otras áreas, especialmente la emocional.

La Figura 2, analiza dentro de la categoría patológico, la relación entre los factores psicopatológicos y las áreas de funcionamiento social de los niños con hemofilia. Se observa que la tendencia a la agresividad se pone de manifiesto en las áreas familiar y emocional. La depresión/ ansiedad aparece notablemente en las áreas emocional y escolar. El rechazo por los iguales predomina en las áreas de relación y emocional.

La Figura 3, estudia la relación de los factores mencionados anteriormente entre los niños hemofílicos ubicados en la categoría dudoso, mostrando el predominio importante de la agresividad en las áreas familiar, escolar y emocional, la depresión/ansiedad resalta en las áreas familiar y emocional. El rechazo por los iguales se hace evidente en el área familiar y en la de relación.

\section{DisCUSIÓN}

Es la primera vez que esta encuesta se utiliza en niños con diagnóstico de enfermedad orgánica crónica, como lo es la hemofilia. Este instrumento fue aplicado en un estudio realizado en niños con depresión que asistían a la consulta externa del Hospital Psiquiátrico de la ciudad de Maracaibo (Rincón, MD; Tesis Doctoral, Universidad del Zulia 1993).

La hemofilia es una enfermedad crónica, con una alta carga de morbilidad que se correlaciona con la severidad de la enfermedad ${ }^{11}$, las secuelas físicas afectan las actividades diarias o son causa de períodos repetidos de hospitalización o visitas ambulatorias frecuentes, además de la necesidad de múltiples cuidados diarios y no poder participar en las actividades con sus compañeros, ocasionando dificultad en su crecimiento y desarmollo integral ${ }^{12}$.

En el presente trabajo se evidenció que la mayoría (56\%) de los niños con hemofilia estuvieron en las categorías de salud mental patológico o dudoso. Este dato resalta la afirmación de que un porcentaje notable de niños que padecen una enfermedad crónica, se enfrentan a numerosos factores de riesgo psicosociales desde muy pequeños y se hacen vulnerables a los mismos, aunque un estudio realizado en los niños hemofílicos atendidos en un centro hospitalario, no mostraron mayores alteraciones con respecto a los niños diabéticos y a los aparentemente saludables, a

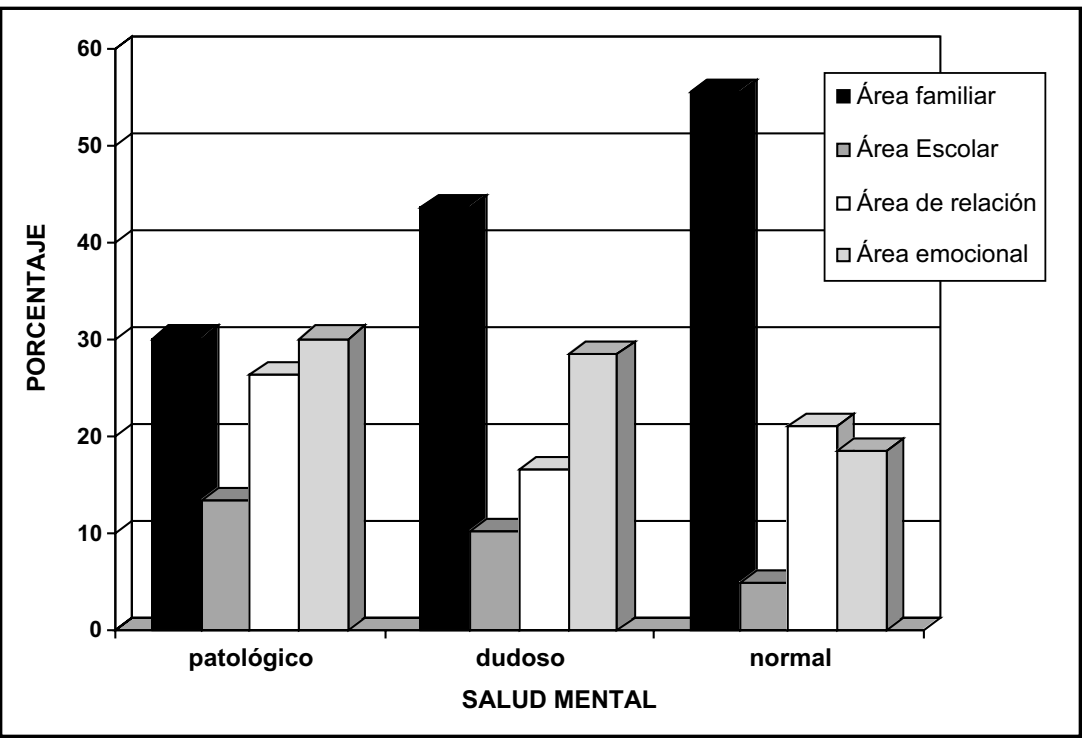

Figura 1. Categorías de salud mental relacionadas con las áreas de funcionamiento social según la encuesta de salud mental «omingo». 
pesar de tener algunos de los primeros diagnóstico de infección con $\mathrm{VIH}^{13}$.

La severidad de la enfermedad, el deterioro de las articulaciones y la incapacidad son parámetros para definir antecedentes de bienestar tanto físico como mental y forman parte de la evaluación de un estudio realizado para establecer las consecuencias de la hemofilia ${ }^{14}$. En este sentido, al relacionar el grado de severidad de la hemofilia con los estados de salud mental, se observó que en la categoría normal predominaron los casos de hemofilia leve y moderada (12/15 pacientes),

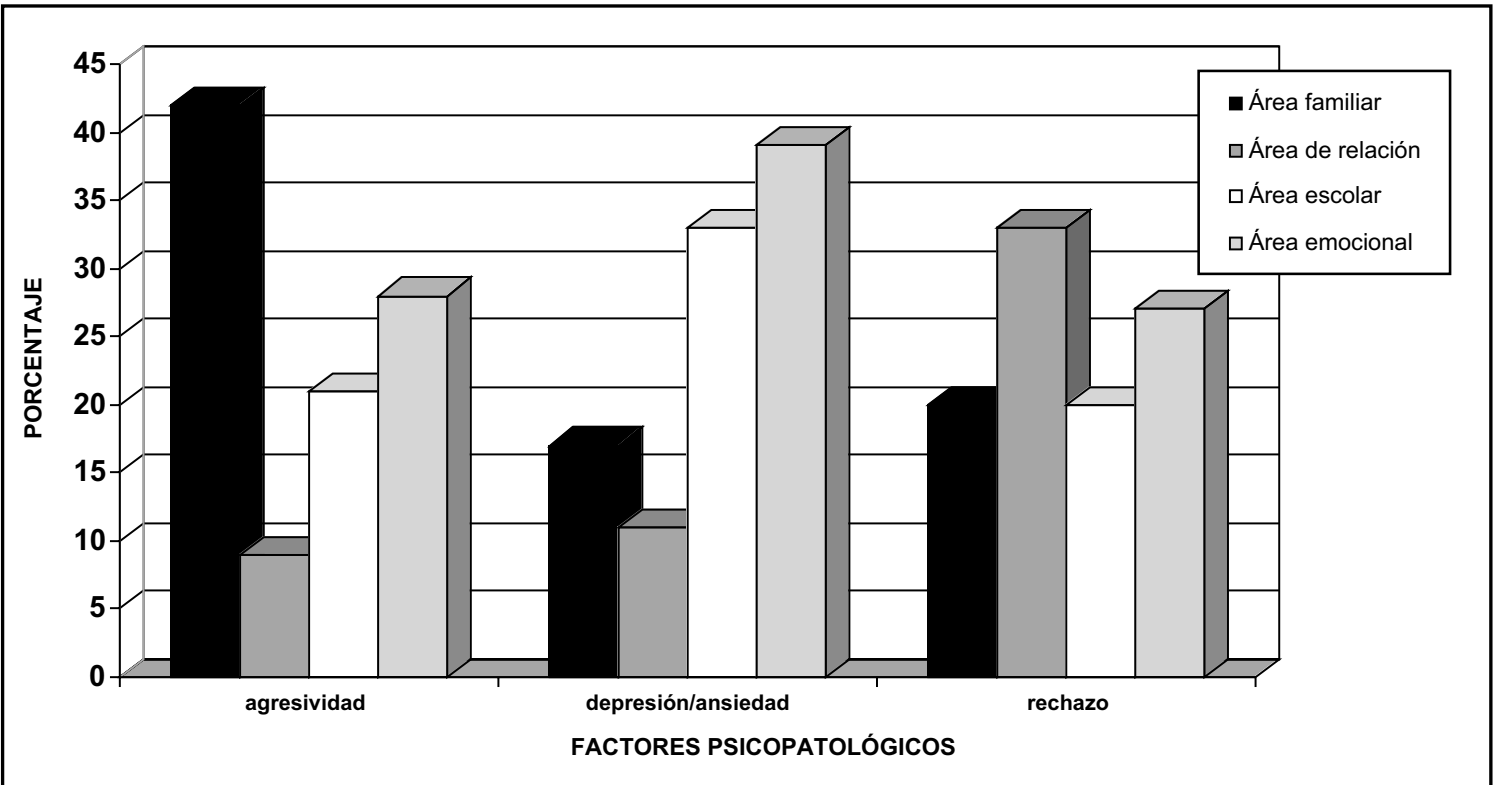

FiguRA 2. Porcentaje de relación entre factores psicopatológicos y áreas de funcionamiento social en niños con hemofilia según la categoría de salud mental patológico.

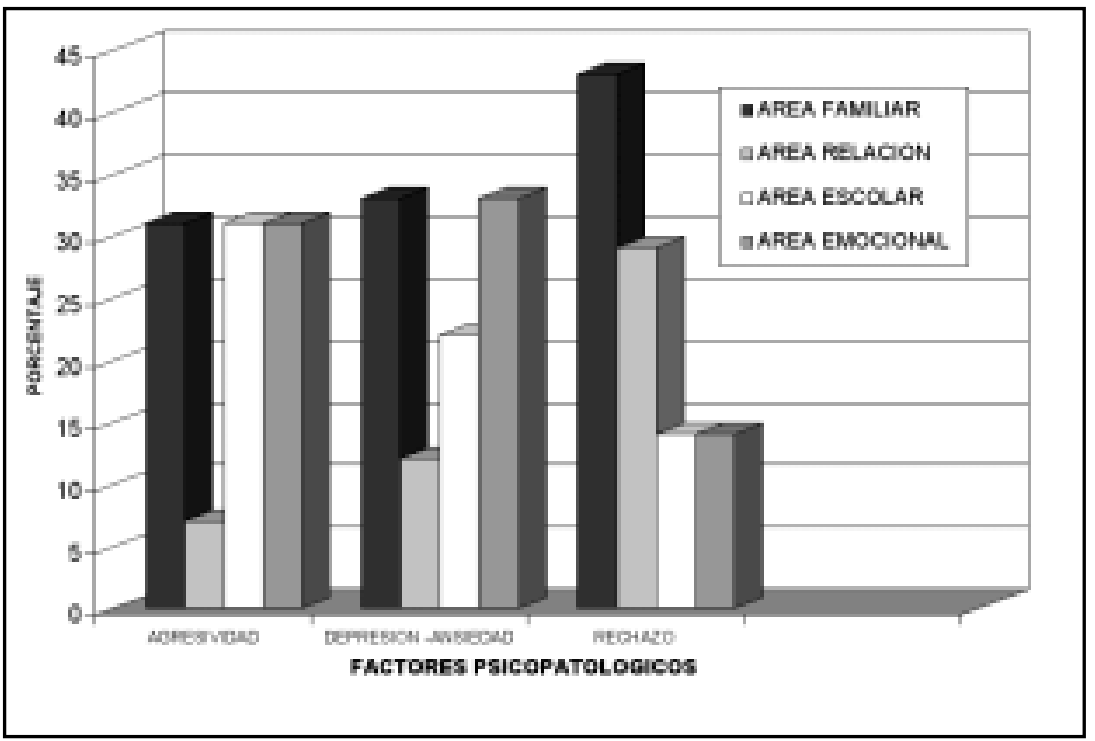

FIguRA 3. Porcentaje de relación entre factores psicopatológicos y áreas de funcionamiento social en niños con hemofilia. Según la categoría de salud mental dudoso. 
mientras que el riesgo de salud mental (patológico y dudoso) se ubicó en los niños con hemofilia moderada y severa (14/19 pacientes). Esto pudiera sugerir que el funcionamiento social de los hemofílicos está probablemente relacionado con el grado de clínico de intensidad de la hemofilia ${ }^{11}$.

En los niños se estudia su funcionamiento social (adaptación o no de su comportamiento), tanto por su respuesta emocional individual como por los aspectos familiar, escolar y social. Al evaluar el funcionamiento de los niños hemofílicos, que resultaron patológicos y dudosos según la ESMD, se evidenció la desadaptación de su comportamiento familiar, emocional y de relación, lo que indica que es en estas áreas donde existe la mayor confrontación del niño con su ambiente externo. De esta manera, en esta investigación se discriminó mejor el riesgo de salud mental de los niños hemofílicos entre los casos de categoría normal y aquellos agrupados en las categorías dudoso y patológico.

Se consideran tres grandes tipos de riesgos psicosociales en el niño con hemofilia: riesgos personales, familiares y socio-educativos. Los riesgos personales son aquellos inherentes al propio comportamiento y personalidad del niño hemofílico. Los riesgos familiares son aquellos inherentes a las pautas de educación familiar. La sobreprotección, la excesiva permisividad, las normas educativas inconsistentes, el culpabilizar y las excesivas limitaciones, generan vulnerabilidad psicológica, en consecuencia los niños no adquieren confianza y autolimitan sus posibilidades de exploración y desarrollo ${ }^{15}$. Entre los riesgos sociales y educativos, el tener amigos y compartir con ellos juegos y experiencias sociales, es uno de los nutrientes fundamentales que los niños tienen para el desarrollo de su personalidad. En un estudio realizado en donde se compararon dos grupos de niños, hemofílicos y escolares aparentemente sanos, se identificaron más problemas en el grupo de los hemofílicos, es decir mayores dificultades emocionales, familiares y de conducta ${ }^{16}$. El mismo estudio permitió evaluar el estrés de los padres de niños hemofílicos y se demostró que el padre presta mayor atención a los problemas de sus hijos y que la madre resultó ser más deprimida y ansiosa acerca de la enfermedad de sus hijos, altamente relacionada con el sentimiento de cul$\mathrm{pa}^{16}$
Al relacionar los factores psicopatológicos en las diferentes áreas de funcionamiento social establecidas en la ESMD, la agresividad se hizo presente en $50 \%$ de la muestra para la categoría patológico y en $57 \%$ de la categoría dudoso, afectando notoriamente el área familiar. Así mismo, la depresión/ansiedad afectó en gran forma las áreas emocional y escolar en estos grupos. Todo parece indicar, a excepción del rechazo social por sus iguales, donde predominó el área de relación, que los factores de riesgo están presentes fundamentalmente dentro del núcleo familiar. Se sabe que existe una sobrecarga hasta un colapso de los recursos emocionales de una familia con un niño crónicamente enfermo. En base a los resultados, una hipótesis a plantear es que la agresividad se expresa en los menos enfermos en tanto los más graves están ansiosos y deprimidos. La forma de abordar los problemas de salud mental del niño hemofílico, sería a través de una integración de él, sus padres y el equipo multidisciplinario de profesionales que atienden esta enfermedad.

Aproximadamente $5 \%$ de los niños y adolescentes de la población general padece de depresión en algún momento ${ }^{6}$. La depresión representa un impacto creciente en los niños y en las familias. Se menciona que $85 \%$ de ellos viven en países en desarrollo y constituyen algunos casos hasta $50 \%$ de la población infantil. Los datos de salud mental en niños y adolescentes señalan que $20 \%$ de los niños sufren trastornos mentales de moderados a severos que comprometen su bienestar y capacidad funcional en la sociedad ${ }^{17}$.

Una investigación realizada en niños hemofílicos encontró que 36\% tenía habilidades sociales pobres: dificultad para comunicar sus necesidades y preocupaciones, para empezar relaciones con el grupo de iguales y para manejar situaciones de estrés. La mayoría mostró comportamientos extremos: pasivos o agresivos y sus conclusiones se basaron en que estas dificultades pueden estar más relacionadas con los modelos educativos seguidos que con la hemofilia en sí18.

Este estudio permitió evaluar el riesgo de salud mental al cual están expuestos estos niños hemofílicos. Por ser un estudio exploratorio no se pudieron extraer conclusiones definitivas, pero sugiere seguir investigando en esta línea donde la salud mental se marca como un factor relevante. 
Ante estos resultados se propone un esquema que abarque una evaluación integral en el aspecto médico y psicosocial del niño hemofílico, su familia y la escuela, para lograr al igual que en otros países, un incremento en la calidad de vida, disminuyendo la tasa de hospitalizaciones y el

\section{REFERENCIAS}

1. Federación Mundial de la Hemofila. Programas. Disponible en: www.wfh.org. Consultado el 20 de mayo de 2004.

2. Ljung R. Paediatric care of child with haemophilia. Haemophilia 2002; 8: 78-82.

3. Ciemente C, Tsiantis J, Sadowski H, Lee C, Baharaki S, BA G ET AL. Psychopathology in children from families with blood disorders: a cross-national survey. Eur Child Adolesc Psychiatry 2002; 11: 151-61.

4. Trzepacz AM, Vannatta $K$, Davies WH, Sthebens Ja, NoL RB. Social, emotional and behavioral functioning of children with hemophilia. J Dev Behav Pediatr 2003; 24: 225-32.

5. Saviolo-Negrin N, Cristante F, Zanon E, Canelini M, Stocco D, Girolami A. Psychological aspects and coping of parents with a haemophilic child: a quantitative approach. Haemophilia 1999; 5: 638.

6. CASEY RL, Brown RT. Psychological aspects of hematologic diseases. Child Adolesc Psychiatr Clin N Am 2003; 12: 567-84.

7. Royal S, Schramm $W$, Berntorp E, Giangrande $P$, Gringeri A, Ludlam C et al. Quality-of-life differences between prophilactic and on-demand factor replacement therapy in European haemophilic patients. Haemophilia 2002; 8: 44-50.

8. Aznar Ja, Magallon M, Querol F, Gorina E, TuseLL JM. The orthopaedic status of severe haemophiliac in Spain. Haemophilia 2000; 6: 170-6.

9. Shapiro $A D$, Donfeeld $\mathrm{SM}$, Lynn HS, Cool VA, StheBEns JA, HunsBerger SL et al. Defining the impact of hemophilia: the academic achievement in children with hemophilia. Pediatrics 2001; 108: E105. número de complicaciones ${ }^{19}$. El objetivo primordial debe corresponderse con la necesidad de cada niño a desarrollar un sistema de vida emocionalmente saludable ante la enfermedad y sus efectos, comprometiendo el apoyo y refuerzo de las organizaciones locales y nacionales de la hemofilia.

10. VALÁs JP. Encuesta de Salud Mental Domingo. Instituto Nacional de Psiquiatría Infantil (INAPSI). Caracas. Venezuela, 1989.

11. Barr RD, Saleh M, Furlong W, Horsman J, Sek J, Pai $M$ ET AL. Health status and health-related quality of life associated with hemophilia. Am J Hematol 2002; 7: 152-60.

12. JoNES P. Living with Heamophilia. Fifth edition. USA. Edi. Oxford University Press, 2004; 352. Disponible en: www.oup-usa.org. Consultado el 20 de junio de 2004.

13. Logan FA, Maciean A, Howie CA, Gibson B, Hann IM, PARRY-Jones WL. Psychological disturbance in Children with haemophilia. BMJ 1990; 301: 1253-6.

14. Arranz P, Costa M, Bayes R, Cancio H, Magalon M, HERNÁNDEZ F. El apoyo emocional en hemofilia. Madrid-España: Editorial Pentacrom. 1996; 29.

15. RITTERMAN MK. Hemophilia context: adjunctive hipnosis for families with hemophiliac member. Fam Process 1982; 21: 469-76.

16. Evans M, Cottreil D, Shiach C. Emotional, behavioural problems and family functioning in children with hemophilia: a cross-sectional survey. Child Care Health Dev 1993; 19: 261-73.

17. Triemstra AH, Van Der Ploeg HM, Smit C, Briet E, ADER HJ, RosendaAl FR. Well-being of hemophilia patients: a model for direct and indirect effects of medical parameters on the physical and psychosocial functioning. Soc Sci Med 1998; 47: 581-93.

18. Celiker R, Kutsal yg, Oy B, Onur O, Gurgey A. Depression in children with hemophilic arthropathy and poliomyelitis: a preliminary report. Turk J Pediatr 2000; 42: 27-30.

19. Fontes EM, Amorim L, Carvalho SM, Farah MB. Hemophilia care in the state of Rio de Janeiro, Brazil. Rev Panam Salud Pública 2003; 13: 1248. 